\title{
PARAMETRIC DESIGN OF DELTA ROBOT
}

\section{Mert Gürgen', Cenk Eryılmaz², Vasfi Emre Ömürlü ${ }^{3}$}

\begin{abstract}
This article describes a sophisticated determination and presentation of a workspace volume for a delta robot, with consideration of its kinematic behavior. With the help of theoretical equations, optimization is performed with the aid of the stiffness and dexterity analysis. Theoretical substructure is coded in Matlab and three-dimensional (3D) data for delta robot are developed in computer-aided design (CAD) environment. In later stages of the project, both 3D and theoretical data are linked together and thus, with the changing design parameter of the robot itself, the Solidworks CAD output adapts and regenerates output with a new set of parameters. To achieve an optimum workspace volume with predefined parameters, a different set of robot parameters are iterated through design optimization in Matlab, and the delta robot design is finalized and illustrated in the 3D CAD environment, Solidworks. This study provides a technical solution to accomplish a generic delta robot with optimized workspace volume.
\end{abstract}

UDC Classification: 62-1/-9 DOI: http://dx.doi.org/10.12955/cbup.v4.856

Keywords: Parallel robot, workspace, dynamic analysis, kinematic analysis, matlab, delta robot.

\section{Introduction}

Especially in a highly competitive products market, organizations are required to manage their manufacturing processes for high efficiency improvements within a short period of time. Those adopting such processes are realizing significant benefits in robotic pick and place applications. Therefore, delta robots provide the flexibility and reliability needed for organizations to compete.

Delta parallel industrial robot is a type of parallel connection robot. Because of its compact structure, large rigid load, and less accumulative error of the joints, it is one of the most widely used robots. Within its mechanical structure, a fixed platform, three input links, and a moving platform are connected together by three linkages composed of four parallel links. Due to the top link being parallel to the bottom link in the four parallel linkages, the moving platform is always parallel to the fixed platform, which is secured by three parallel linkages. These provide three-degrees-of-freedom (3DOF) movement capability within a predefined area, with the help of its unique mechanical structure. Because of the parallel architecture, there is no cumulative deviation on any axis. Therefore, the delta robot becomes inexpensive and, with high acceleration capability of the packaging system for conveyor production lines, is highly preferred (Roy ve \& Olgac, 1997).

This study designs a concept for a delta robot, and presents assumptions in making the new configuration. First, the design for a novel configuration to create a 3DOF translation robot is outlined with a free-body diagram. Next, both forward and inverse kinematic calculations are derived. Then, further optimization of the workspace volume of robot is examined. To clarify, the theoretical background of the robot itself, the Jacobian method is formulated and verified by comparing results with non-optimized parameters. The stiffness and dexterity analysis is derived from the Jacobian method and coded in Matlab in order to find the average for a given workspace. An optimization problem is then devised to maximize the workspace stiffness and minimize dexterity. The optimization is set up in Matlab, and involves creating an objective function to achieve our objective. An optimization stage simply involves inputting data, two link lengths, and iterating those parameters.

\section{The 3DOF Delta Parallel Robot Design}

Parallel robots with 3DOF are parallel manipulators comprised of both a fixed base and a payload platform, linked together by three independent, identical, and open kinematic chains. The delta parallel robot consists of a spatial parallel structure with 3DOF, which is driven by three revolute actuators. The platform is connected to each drive by two links, forming a parallelogram, and this allows only translational movements of the platform, while keeping the platform parallel to the base plane (Verdes, Sergiu-Dan, Milos \& Radu, 2010).

\footnotetext{
${ }^{1}$ Mert Gürgen, Yıldız Technical University, Department of Mechanical Engineering, Turkey, mertgurgen@yahoo.com

${ }^{2}$ Cenk Eryılmaz, Y1ldız Technical University, Department of Mechatronics Engineering, Turkey, cenk.eryilmaz@gmail.com

${ }^{3}$ Vasfi Emre Ömürlü, Yıldız Technical University, Department of Mechatronics Engineering, Turkey, omurlu@ yildiz.edu.tr
} 


\section{Forward Kinematic Analysis}

Figure 1: Delta robot geometric parameters

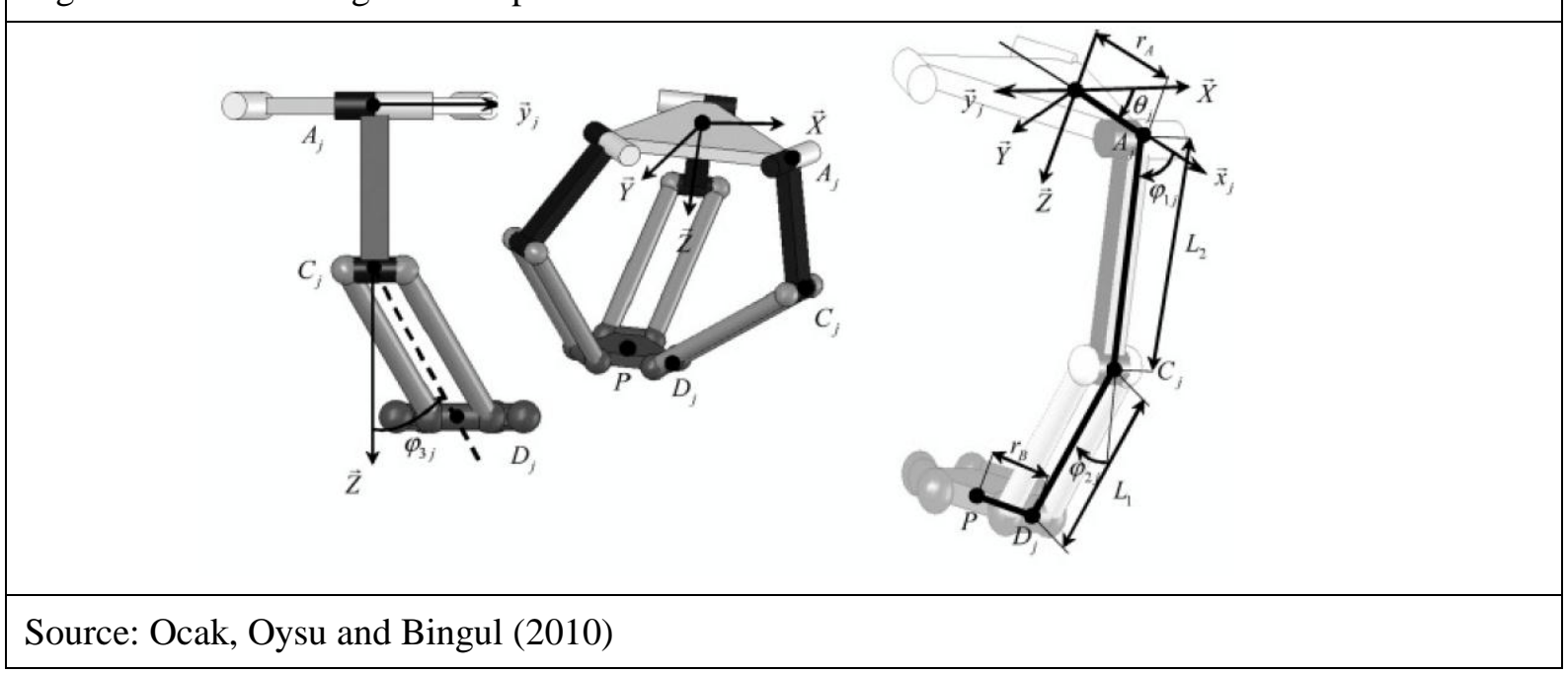

The constraint equations were generated by applying Pythagoras' rule in three dimensions to each pair of arms. The Delta robot geometry parameters, as shown in Figure 1: $L 1, L 2, r A, r B, \theta j(j=1,2,3)$, with $\phi 1, \phi 2, \phi 3$ defining the joint angles (Ocak, Oysu \& Bingul, 2010) and $\mathrm{P}$ is the point on a moving plate. The geometric model of this point can be expressed by the parameters in Figure 1.

\section{Inverse Kinematic Analysis}

The unknown values, the joint angles, were calculated using the P point, which was determined in the forward kinematic phase. Inverse kinematic relationships for the delta robot are relatively simple. The most computationally efficient method of calculating the Jacobian matrix was used to differentiate kinematic relationships with respect to the $3 \mathrm{D}$ coordinates of the tip (x, y, and $\mathrm{z})$. This yielded the elements for the inverse Jacobian and inverted matrix.

\section{Jacobian, Stiffness and Dexterity Analysis}

The Jacobian of a parallel robot is related mathematically to the velocity of the end effector and the actuated joints. To calculate the Jacobian, the values of $\theta 1, \theta 2$, and $\theta 3$ were used from the inverse kinematics. The Jacobian is defined as $A x^{\prime}=B p^{\prime}$ where $x^{\prime}$ is the Cartesian rate of change and $p^{\prime}$ is the actuator rate of change. By using the Jacobian matrix or even the stiffness matrix, it was possible to calculate the dexterity of the robot, which is another important kinematic factor that can affect the pose accuracy of the parallel robot (Merlet, 2006). The simplified mechanical structure, with the required parameters, is given in Figure 2. It is possible to find a solution to the Jacobian equation by solving three equations, for the three unknowns. Once obtained, the Jacobian calculations were converted into a suitable format for Matlab, based on the link lengths for the robot and the joint angles for the given point in the workspace.

\section{Optimization Stage of Delta Robot}

The aim of this section was to develop and solve the multidimensional, non-linear optimization problem, while selecting geometric design variables that provide an acceptable compromise between manipulability and space utilization. The optimization considered the effect of the design variables on a utility function containing two performance indices, each calculated by analyzing an arbitrary cross section of the workspace. A numerical optimization was carried out in the Matlab environment, and the formulation and results of this optimization are presented in the following sections.

By using the eigenvalues of the stiffness matrix, we avoided having to calculate the inverse of the Jacobian, which saved valuable time during optimization. Like the stiffness, the dexterity for all reachable points in a workspace was averaged to obtain a single dexterity value for input in optimizing the workspace. The dexterity analysis defined the accessibility and steady points on the Z-axis for the delta robot. In this way, a delta robot arm length can be chosen (Table 1), based on the accessibility and steady points on the Z-axis (Dogan, 2010). 
Figure 2: Schematic labeling for Jacobian

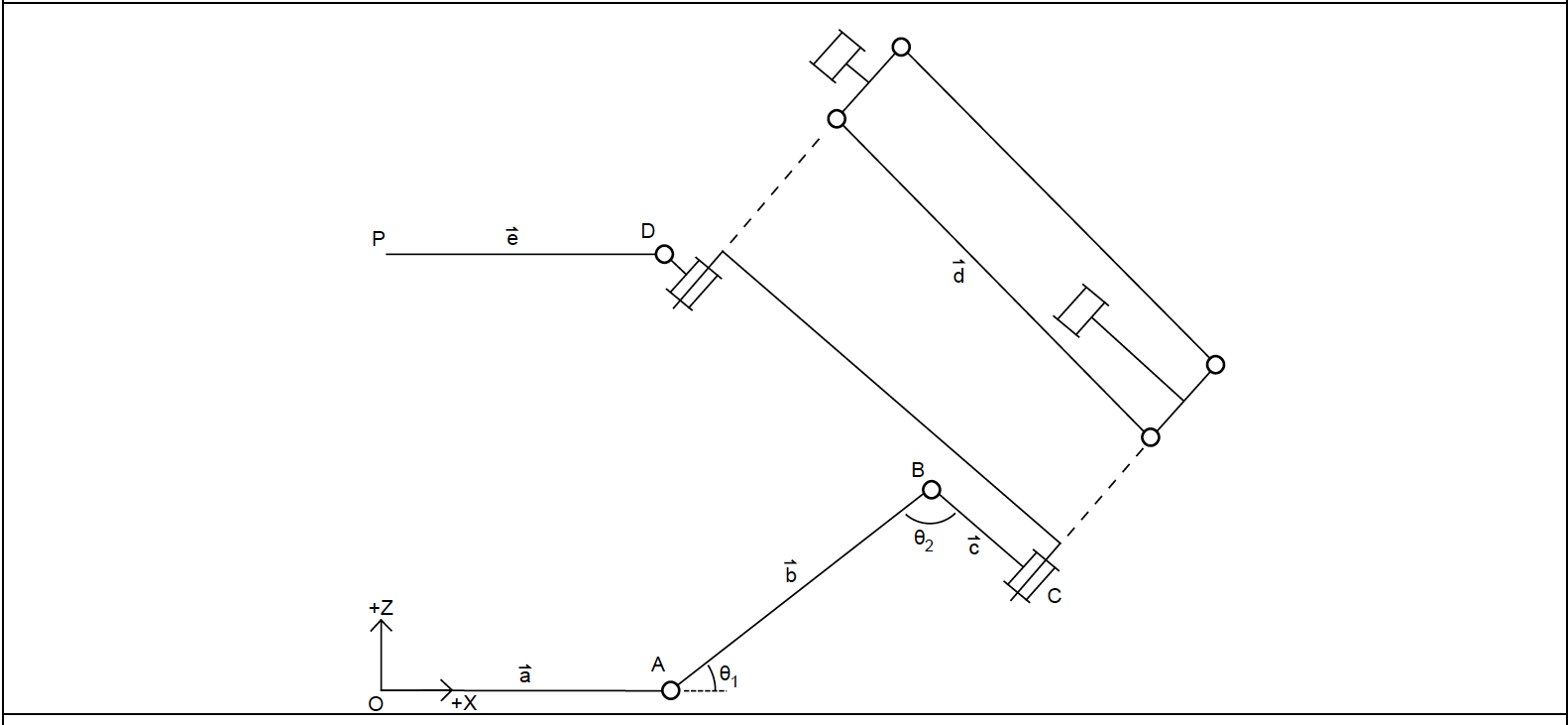

Source: Author

Table 1: Parameters of delta robots

\begin{tabular}{|c|r|r|r|r|c|}
\hline Optimization Type & $\begin{array}{c}\mathbf{L}_{\mathbf{1}} \\
(\mathrm{mm})\end{array}$ & $\begin{array}{c}\mathbf{L}_{\mathbf{2}} \\
(\mathrm{mm})\end{array}$ & $\begin{array}{c}\boldsymbol{r}_{\boldsymbol{A}} \\
(\mathrm{mm})\end{array}$ & $\begin{array}{c}\boldsymbol{r}_{\boldsymbol{B}} \\
(\mathrm{mm})\end{array}$ & $\begin{array}{c}\text { Volume } \\
\left(\mathbf{m m}^{\mathbf{3}}\right)\end{array}$ \\
\hline $\mathbf{1}$ & 50 & 90 & 50 & 60 & $1.69 \times 10^{6}$ \\
\hline $\mathbf{2}$ & 30 & 70 & 50 & 60 & $1.96 \times 10^{6}$ \\
\hline $\mathbf{3}$ & 40 & 80 & 50 & 60 & $2.04 \times 10^{6}$ \\
\hline
\end{tabular}

$\mathrm{L}_{1}=$ top arm length; $r_{1}=$ top table radius, $\mathrm{L}_{2}=$ bottom arm length; $r_{2}=$ bottom table radius

Source: Author

\section{Optimization Result}

In this optimization study, the workspace volume was increased by varying the arm lengths of the delta robot. The optimization stage was aided with kinematic solutions (including Jacobian and the stiffness and dexterity analysis) to achieve greater efficiency from the iterations. At the end of the optimization stage, the workspace of the delta robot was calculated and illustrated, respectively, as shown in Figure 3.

Figure 3: Optimization of workspace through iteration
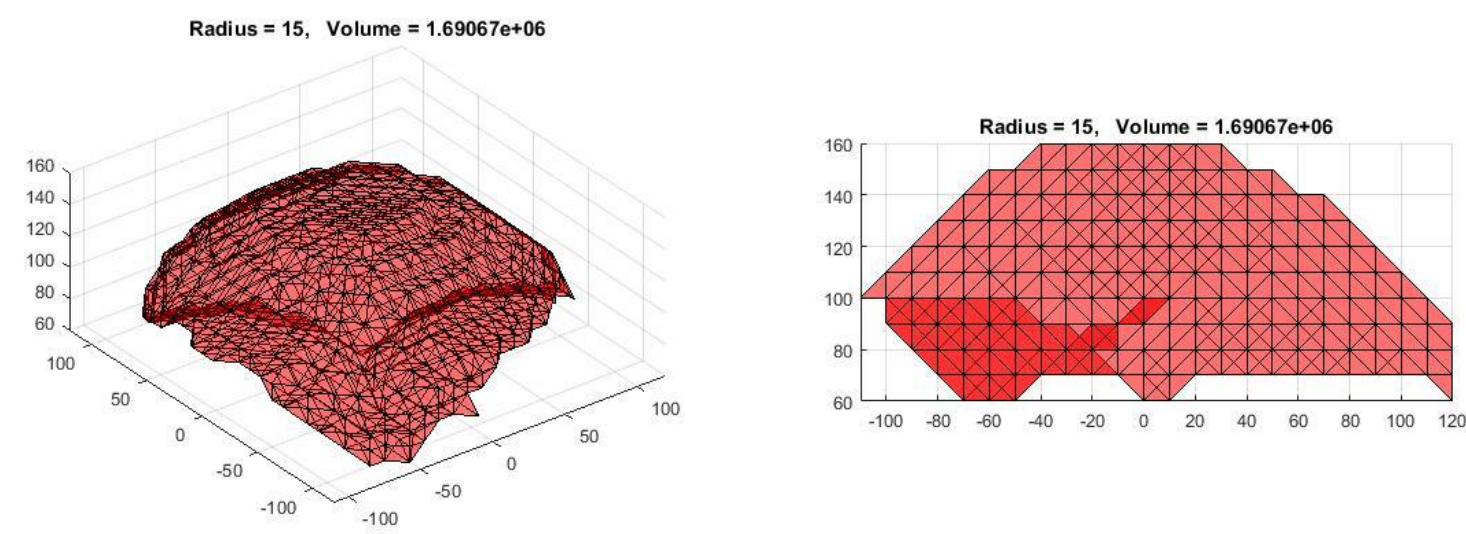

Iteration result (1): Perspective and X-Z section view, respectively 


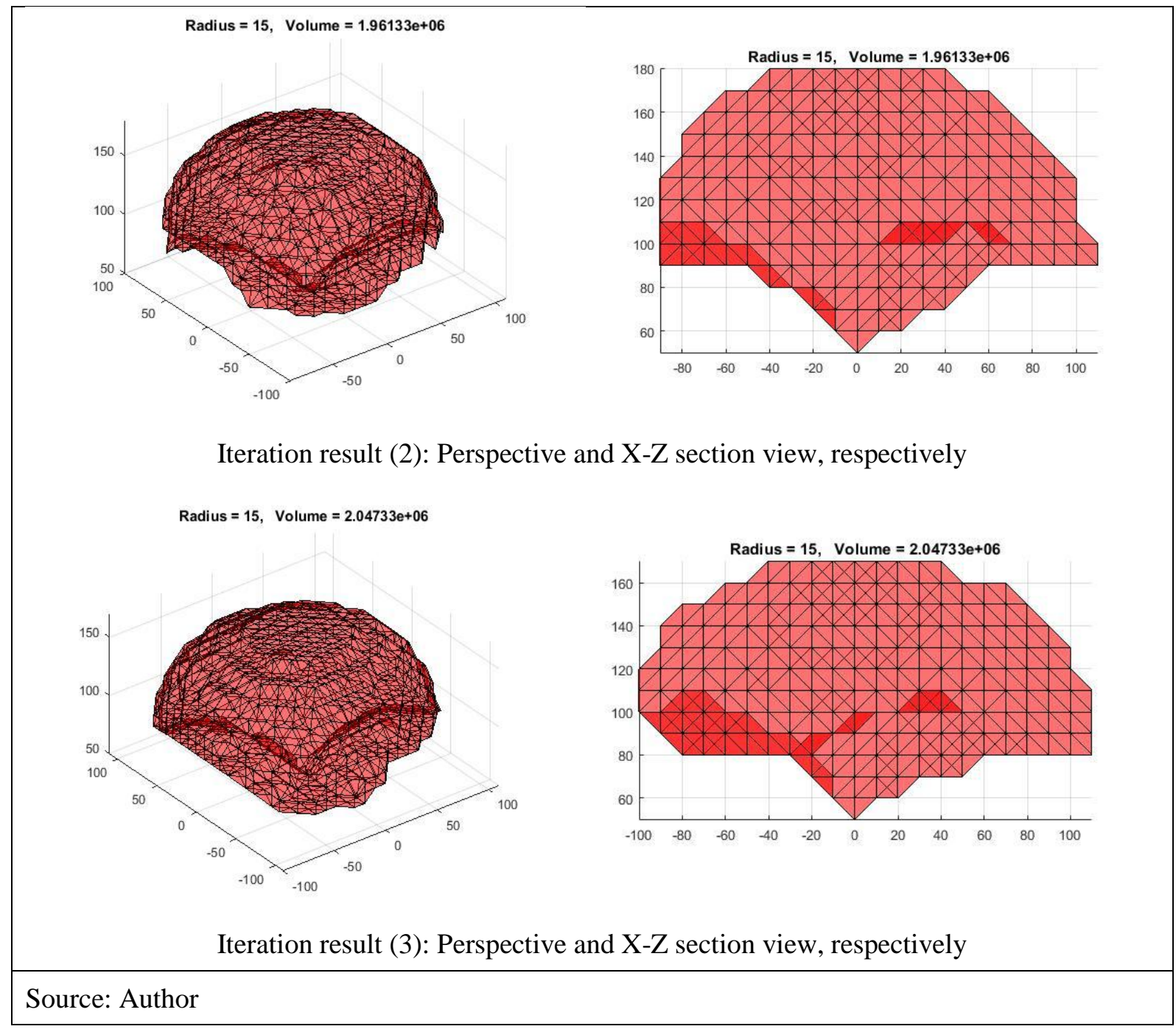

\section{Conclusion}

This study demonstrated a theoretical solution for deriving an optimum workspace for a delta robot with use of different sets of arm length and plate diameter values, to achieve robot accessibility within a 3D coordinate system. Theoretical calculations included a stiffness and dexterity analysis, combined with the Jacobian method, and from this, iterations were finalized with the workspace volume illustrated. This study's findings could be used to define a set of delta robot link/plate parameters for use in applications for improving efficiency in organizations required to manage their manufacturing processes in a competitive products market.

\section{References}

Dogan, E. (2010). Calculations of Delta Parallel Robots Forward and Inverse Kinematics. Conference on Eskisehir Osmangazi University. 61-65.

Merlet, J. P. (2006). Jacobian, manipulability, condition number, and accuracy of parallel robots. Journal of Mechanical Design. 128(1):199-206.

Ocak, O., Oysu, C., \& Bingul, Z. (2010). Design and Simulation of Delta Robot. Proc. of the Conference on Automatic Control. 1-6.

Roy ve, R. G. \& Olgac, N. (1997). Robust Nonlinear Control via Moving Sliding Surfaces. Proc. of the 36th Conference on Decision and Control. 943-948.

Verdes, D., Sergiu-Dan, S., Milos, M., \& Radu, B. (2010). Mechatronic design, kinematics analysis of a 3 DOF medical parallel robot. IEEE-ISRCS'10, the 3rd IEEE Symposium on Resilience Control Systems. 3-4. 